\title{
Cyclostationary Signatures for Rendezvous in OFDM-based Dynamic Spectrum Access Networks
}

\author{
Sutton P.D., Nolan K.E., Doyle L.E. \\ Centre for Telecommunications Value-Chain Research (CTVR) \\ University of Dublin, Trinity College, Ireland \\ Email: suttonpd@tcd.ie, keith.nolan@ctvr.ie, ledoyle@tcd.ie
}

\begin{abstract}
Distributed coordination of operating frequencies and bandwidths is one of the key challenges in the development of dynamic spectrum access (DSA) technology. The spectrum efficiency of DSA networks is achieved through the use of spectrum white spaces, frequency bands which are unoccupied at a given time and place. The issue of frequency rendezvous arises as the availability of these white spaces may change dynamically in frequency, time and space.

We propose a novel solution to the problem of distributed rendezvous in dynamic spectrum access networks using cyclostationary signatures. By embedding a cyclostationary signature or watermark in a transmitted signal, the received signal can be uniquely detected, classified, and used for frequency acquisition by distributed dynamic spectrum access-enabled nodes in a network. This paper therefore makes three key contributions. First, a solution to the problem of distributed rendezvous in DSA networks using the novel concept of cyclostationary signatures is presented. Second, a low-complexity technique for generating these cyclostationary signatures in OFDM (orthogonal frequency division multiplexing) waveforms is described. Finally, an initial performance analysis of an implemented test platform is discussed.
\end{abstract}

\section{INTRODUCTION}

The current command and control approach used for frequency spectrum allocation and assignation by spectrum regulators to operators has led to a perceived shortage of spectrum for new wireless services. In fact, recent studies [1] have shown that although most of the useful frequency spectrum is allocated, only a fraction of it may be in use at any given time or place. By filling the unoccupied portions of spectrum or white spaces and using spectrum resources more efficiently it may be possible to introduce new price models, create a market place for buying, selling, exchanging and leasing spectrum, and encourage the development and deployment of new innovative wireless systems and services.

Dynamic spectrum access (DSA) technology offers a solution to the current spectrum usage inefficiencies based on the ability to dynamically adapt operating frequencies and bandwidths to occupy spectrum white spaces. A number of significant challenges must be overcome before this solution can be realized however. One of these key challenges is that of frequency rendezvous. In traditional wireless communications networks, devices typically have a priori knowledge of the initial operating frequencies to be used. This means that upon commencing operation, nodes within the network may have a predetermined frequency, or list of frequencies which can searched in an attempt to established a wireless communications link with their peers. In a DSA network, the frequency of operation may not be known initially; establishing a common communications channel may be dependent on the available spectrum (the white space spectrum). The potential channel may therefore lie within a much greater frequency range and may also change during the operating lifetime of the network. The challenge of frequency rendezvous in this case involves not only synchronization with, but also the detection and classification of signals of peer devices in the network.

The use of a common control channel has been suggested in previous research work [3] in order to overcome the issue of frequency rendezvous. However, in a DSA network environment, the spectrum available for use may change dynamically. As a result, it may not be possible to allocate a fixed control channel and a distributed solution must therefore be devised. Additionally, a common control channel implies the specification and support of predefined protocols by all systems wishing to use the spectrum band in question. By minimizing such requirements in the implementation of a practical DSA approach, the range of systems capable of using the band may be kept to a maximum.

The solution proposed involves the use of cyclostationary signal analysis, a powerful tool for the detection and classification of communications signals. Many of the waveforms used in wireless telecommunications exhibit periodicities of their second order statistics due to the coupling of message signals with periodic signals such as sinusoidal carriers, pilot sequences, spreading codes and repeating preambles. These periodicities often result in specific patterns of correlation in the spectral properties of the signal which may be detected even at low signal to noise ratios (SNR) and used for classification and analysis. Second order cyclostationary signal analysis involves the use of appropriate non-linear transformations to expose and examine these patterns [4].

In order to leverage the benefits of cyclostationary signal analysis for rendezvous in DSA networks, we propose the use of Orthogonal Frequency Division Multiplexing (OFDM) as a suitable spectrum access scheme. An OFDM signal is generated in the frequency domain using an inverse fast Fourier transform (IFFT). This OFDM signal can be 'sculpted' to create different power spectrum profiles by manipulating the frequency bins used to create the signal. As the power spectrum can be dynamically changed according to the varying 
characteristics of the frequency spectrum, OFDM is particularly suited for use in DSA networks.

The flexibility of OFDM creates the potential for unique signal identifiers with a high probability of detection even at low signal to noise ratios (SNR). Specific periodicities or cyclostationary signatures can be embedded in OFDM signals allowing the flexibility of this spectrum access scheme to be combined with the performance of cyclostationary signal analysis. It is this combination that is particularly suited to addressing the challenge of frequency rendezvous in DSA networks.

In Section II, we provide a brief overview of dynamic spectrum access networks, OFDM and cyclostationary signal analysis. The concept of a cyclostationary signature is introduced in Section III. In addition, the proposed technique for generating signatures in OFDM waveforms is presented and the key benefits of using cyclostationary signatures in DSA networks are examined. In Section IV, the implementation of a reconfigurable cyclostationary signature detector on a DSA network test platform is outlined. Section V presents experimental results and Section VI discusses the performance of the experimental testbed and detection scheme. Ongoing and future plans for this technology are outlined and the paper is concluded in Section VII.

\section{BACKGROUND}

\section{A. Dynamic Spectrum Access Networks}

In 2002 the FCC Spectrum Policy Task Force published a report [5] which clearly stated the case for spectrum policy reform on the basis of three principle arguments.

First, it was argued that current spectrum policies are outdated and under strain from the dramatic increase in demand for spectrum-based services and devices. Second, it was acknowledged that technological advances are enabling alternative approaches to spectrum policy. As communications systems become increasingly sophisticated and the manner in which they are capable of using spectrum becomes more flexible, the policies which govern their operation may also become more flexible. Third, it was stated that the lack of availability of spectrum for new services or for expansion of existing ones was not a result of heavy utilization but rather extremely inefficient usage due to inflexible spectrum policies. This argument has been strengthened by recent studies [1] illustrating the low levels of utilization of much of the allocated spectrum.

The potential benefits of overcoming the issue of spectrum scarcity are considerable. By developing wireless systems capable of using spectrum more efficiently and introducing more flexible approaches to spectrum management, spectrum access for new and existing systems and services may be made readily available. Increased availability of spectrum would reduce entry and overhead costs for systems and services and further encourage the type of innovation already seen in unlicensed spectrum bands. In addition, increased exposure to competition and market forces may be used to ensure that the maximum benefit is derived from the spectrum resources available.

It is important to note that a solution to the problem of spectrum scarcity may not be achieved by either spectrum policy reform or technological advances alone. Rather, approaches in which the two are developed side-by-side are required. Thus, the term Dynamic Spectrum Access (DSA) Network is used to describe a wireless system designed to efficiently utilize radio spectrum through the exploitation of increasingly flexible spectrum management regimes.

Broadly speaking, such proposed systems fall into one of two categories. The first of these is referred to as the licensed or property rights model. Under this approach, users are assigned exclusive-use licenses, affording them the flexibility to decide the manner in which the spectrum is used including the technology employed and services provided. Market forces may be introduced by permitting the trade of these licenses in secondary markets. In addition, licensees may choose to allow third party operators to access their spectrum while avoiding the creation of harmful interference. This form of spectrum access has been referred to as easement usage and generally takes one of two approaches - either underlay or overlay. Overlay spectrum access involves the opportunistic use of spectrum resources which are not in use by the licensee at a given time and place. In contrast, systems employing underlay access avoid the creation of harmful interference by embedding signals in the noise floor observed by licensee devices.

The second broad category of DSA systems is described by the commons model. Rather than granting exclusive usage rights to a single licensee, the commons approach permits shared access to the spectrum resource by a number of users. The approach relies upon an agreed protocol or etiquette for avoiding harmful interference, allocating spectrum in times of congestion and resolving conflicts. Only those users who choose to conform with this protocol are granted access. Thus, decision-making authority is decentralized to the users of the spectrum resource in the commons model. A more thorough examination of both the licensed and commons models may be found at [2].

In examining each of the approaches described above, a number of common technical challenges may be identified. Among these are the issues of sensing, allocation and coordination which arise in both the commons model and the easement usage approaches under the licensed model.

Sensing involves observation of the radio environment to identify spectrum resources which may be used without causing harmful interference to other wireless systems. These unused spectrum resources are often termed white spaces and can vary over time, frequency and space. Allocation concerns the fair distribution of spectrum resources among competing wireless systems. Under the licensed model, pricing systems and market forces determine the distribution of spectrum whereas under the commons model, the protocol adopted is responsible for ensuring fair access. Coordination involves the distributed agreement of operating frequencies and bandwidths 
between nodes within a DSA system. It is the challenge of coordination which is addressed by this paper.

\section{B. Orthogonal Frequency Division Multiplexing}

Orthogonal frequency division multiplexing (OFDM) is a multi-carrier transmission scheme which has significant advantages for high data rate communications in non-line-of-sight applications. The scheme involves the conversion of a high rate data stream into a number of parallel low rate streams which are then mapped onto closely spaced orthogonal carriers. By performing this conversion, a number of benefits are realized.

First, the decrease in symbol rate on each carrier provides a reduced sensitivity to inter-symbol interference. In multipath environments this robustness may be improved through use of a cyclic prefix on each symbol transmitted. The cyclic prefix effectively collects multipath components which arrive within a given period, allowing them to be used to contribute constructively to the received symbol.

A second benefit arises from the flat fading that is experienced at each narrowband carrier. In high bandwidth single carrier transmission schemes, signals are distorted by frequency selective fading which requires complex equalization. By using parallel narrowband carriers, the fading may be treated as flat and may be equalized using much less complex structures.

A third significant benefit of OFDM as a transmission scheme is the availability of an efficient implementation in the form of the inverse fast Fourier transform (IFFT).

Each of these advantages have contributed to the adoption of OFDM as the transmission scheme of choice for a number of recent standards including IEEE 802.11 [6], IEEE 802.16 [7], Digital Audio Broadcasting (DAB) [8], Terrestrial Digital Video Broadcasting (DVB-T) [9] and the first proposed wireless standard based on dynamic spectrum access cognitive radios, IEEE 802.22 [10].

When applied to DSA networks, OFDM provides a considerable advantage over alternative transmission schemes due to the flexible nature of its power spectrum. In using the IFFT to generate an OFDM symbol, a vector of bins is used to represent the parallel carriers in the frequency domain. By altering the contents of these bins, the frequency domain properties of the resultant OFDM symbol are directly impacted. It has been shown that this approach may be used effectively for both interference avoidance [11] and bandwidth matching [12] in DSA applications. Another considerable benefit of using OFDM is the spectral occupancy and channel condition information which may be obtained through the use of an FFT process in the receiver. As an IFFT is used to create the OFDM symbol transmitted, an FFT is performed upon received symbols in order to extract the modulated data. In cases where data transmission is not taking place or where a number of carriers are not in use, the corresponding FFT bins may be used to obtain power spectral density (PSD) information for the channel.

\section{Cyclostationary Signal Analysis}

Many of the digital communications signals in use today exhibit periodicities of their second order statistical parameters due to the coupling of stationary message signals with periodic sine wave carriers, pulse trains or repeating spreading codes and operations such as sampling, scanning, multiplexing and coding. These cyclostationary properties give rise to unique spectral correlation features which may be detected through the use of appropriate nonlinear transformations.

Much of the initial work examining the use of cyclostationary models in the analysis of telecommunication signals was carried out by W.A. Gardner and his colleagues [13] [14] [15]. In [16] a number of significant advantages of using cyclostationary models in the detection and classification of signals in difficult environments were identified. Recently, similar approaches have been suggested as solutions to the challenge of low power signal detection and classification for opportunistic DSA networks [17] [18]. As well as signal detection and classification, cyclostationary signal analysis has been applied to the areas of synchronization [19], blind channel characterization and equalization [20] and frequencyshift filtering [21].

In the analysis of cyclostationary signals, two key functions are typically utilized. Time domain analysis of cyclostationary signals is performed using the Cyclic Autocorrelation Function (CAF),

$$
\hat{R}_{x}^{\alpha}(\tau)=\lim _{\Delta t \rightarrow \infty} \frac{1}{\Delta t} \int_{-\Delta t / 2}^{\Delta t / 2} x(t+\tau / 2) x(t-\tau / 2) e^{-i 2 \pi \alpha t} d t
$$

The frequency domain equivalent of the CAF is its Fourier transform, the Spectral Correlation Density (SCD)

$$
\begin{aligned}
S_{x}^{\alpha}(f)= & \lim _{\Delta f \rightarrow \infty \Delta t \rightarrow \infty} \lim _{\Delta t} \frac{1}{\Delta t} \int_{-\Delta t / 2}^{\Delta t / 2} \Delta f X_{1 / \Delta f}\left(t, f+\frac{\alpha}{2}\right) \\
& \cdot X_{1 / \Delta f}^{*}\left(t, f-\frac{\alpha}{2}\right) d t
\end{aligned}
$$

, where

$$
X_{1 / \Delta f}(t, v)=\int_{t-1 / 2 \Delta f}^{t+1 / 2 \Delta f} x(u) e^{-i 2 \pi v u} d u
$$

is the complex envelope of the narrow-band-pass component of $x(t)$ with centre frequency $v$ and bandwidth $\Delta f$.

For cyclic frequency $\alpha=0$ these reduce to the conventional autocorrelation function and power spectral density function respectively, related by the well-known Wiener-Khinchin theorem [22].

The significant advantages of cyclostationary signal analysis when compared with alternative approaches lie in the wealth of information which may be represented by the SCD of a signal. Although the presence or absence of a given signal may be indicated by the specific cyclostationary features detected, these features may also be used to determine key signal properties such as carrier frequency, keying frequency and 
phase properties. These may then in turn be used for timing and frequency synchronization [23] or for signal classification and discrimination [24].

The unique ability of cyclostationary signal analysis to derive detailed information about signals of interest without requiring phase-related information about those signals make it a very useful tool in solving the issue of frequency rendezvous in DSA networks. In a DSA networking application, an awareness of the radio environment within which a device operates is key. While coherent techniques require close synchronization with signals of interest, cyclostationary signal analysis may be applied to obtain information about signals which are present anywhere within the bandwidth which is sampled. These advantages are further examined and the novel use of cyclostationary signatures to exploit them are presented in the next section.

\section{Cyclostationary Signatures}

In Section II-C it was stated that many digital communications signals may be modeled as cyclostationary random processes as they exhibit periodicities of their second-order statistics. In the frequency domain, these properties manifest themselves as patterns of correlation within the spectrum of those signals.

In this section, the novel approach of artificially creating a specific pattern of spectral correlation within an OFDM signal to form a unique signature or watermark is presented. A number of methods by which this may be achieved are examined and the uses of such an approach in the context of DSA networks are discussed.

\section{A. Signature generation}

A cyclostationary signature is a term used to describe a spectral correlation pattern present in a digital communications signal which may be detected through cyclostationary analysis and used to uniquely identify it.

The spectral correlation properties of telecommunication signals usually reflect the inherent cyclostationary properties of those signals. However, by manipulating the IFFT frequency bins used to generate an OFDM signal, it is possible to artificially introduce correlation patterns in the power spectrum of that signal. These patterns can be used to form a cyclostationary signature or watermark to aid the detection and classification of the transmitted signal.

Figure 1 illustrates one method which may be used to embed a simple cyclostationary signature in an OFDM signal. Figure 1(a) shows the manner in which a number of the IFFT bins used in the generation of the OFDM symbol may be repeated in order to create a spectral correlation pattern. The signature created can be clearly identified in the normalized SCD of the resulting signal shown in Figure 1(b). By viewing the SCD from above, as in Figure 1(c), the spectral and cyclic frequency properties of the generated signature may be identified. In this case, the signature is centred upon the carrier frequency of the OFDM signal due to the equidistance of the mapped OFDM carriers from the DC carrier. The overall distance between the

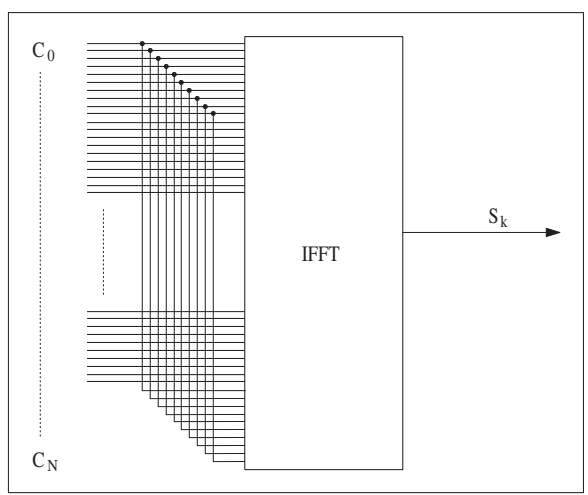

(a) Signature generation

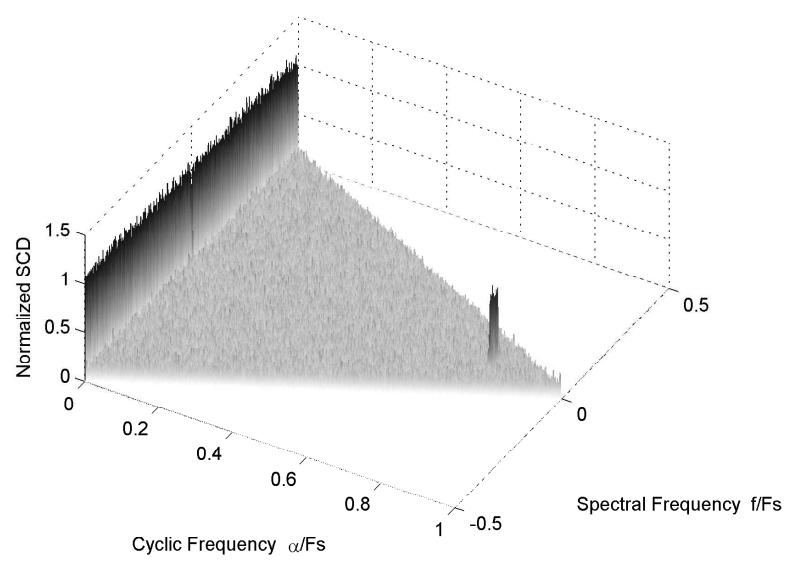

(b) Normalized Spectral Correlation Density

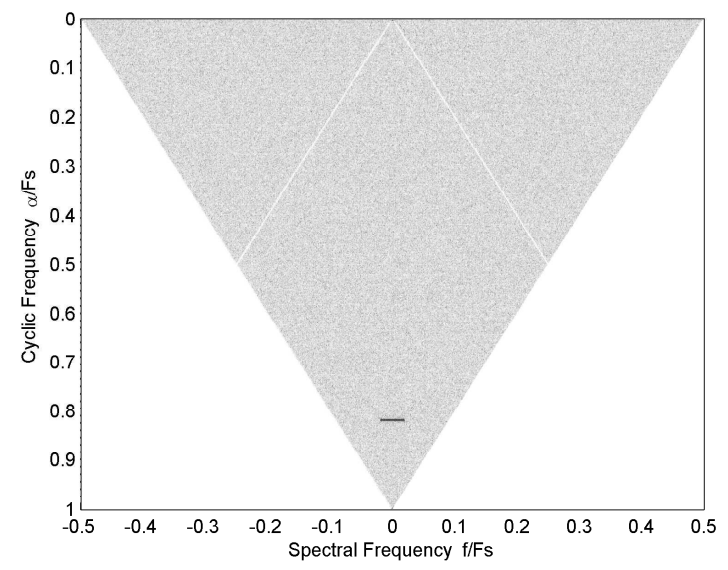

(c) Signature at $\alpha=0.8 F_{s}$

Fig. 1. Creation of a simple cyclostationary signature with OFDM carrier mapping 


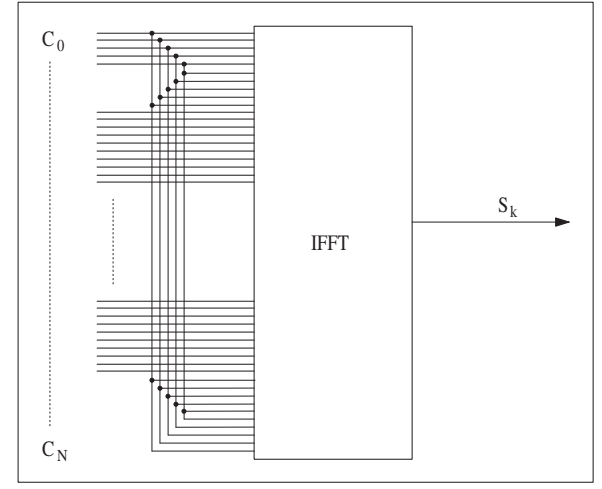

(a) Signature generation

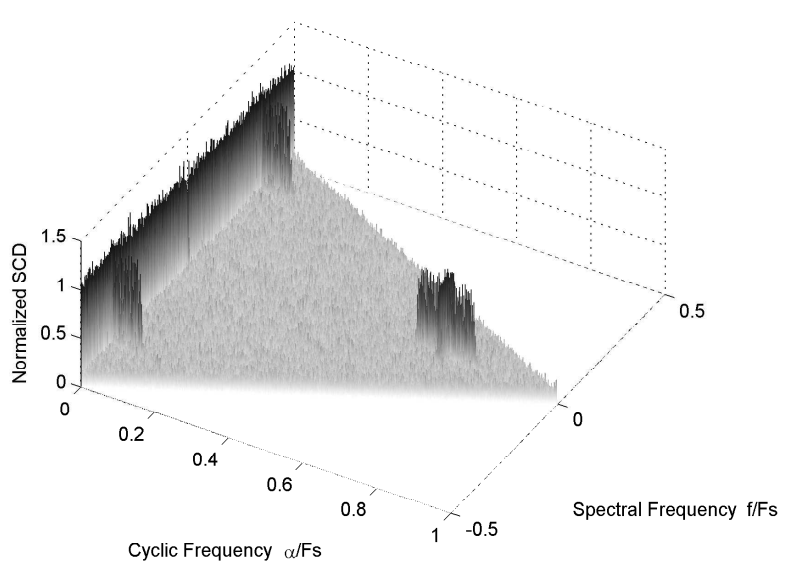

(b) Normalized Spectral Correlation Density

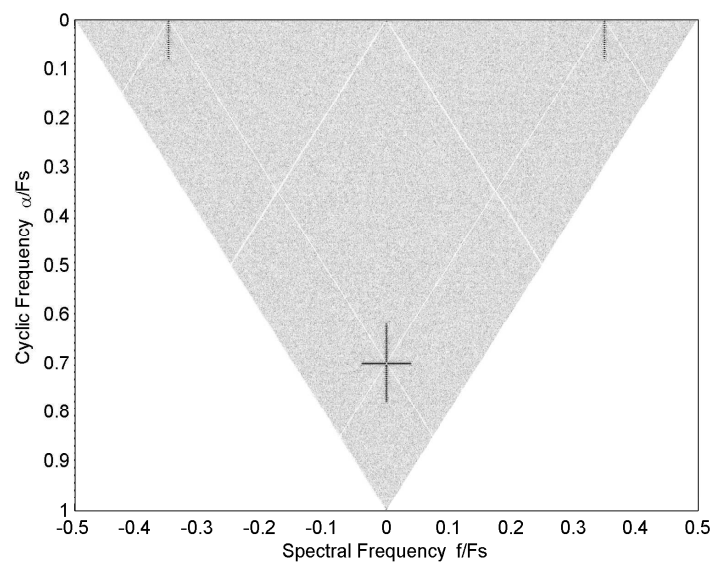

(c) Signature at $\alpha=0.7 F_{s}$

Fig. 2. Creation of a more complex cyclostationary signature mapped carriers determines the cyclic frequency at which the signature may be detected, in this case $\alpha=0.8 F_{s}$.

Figure 2 outlines the creation of a more complex signature using multiple mappings of a number of IFFT bins. This approach results in a signature which contains features over a range of values of $\alpha$ and serves to illustrate the flexibility with which cyclostationary signatures may be generated using the IFFT stage of an OFDM transmission scheme.

One of the advantages of using OFDM carrier mapping to generate cyclostationary signatures is the ability to accurately locate the features of the signature on the spectral and frequency axes of the SCD by carefully choosing the carriers used. For a signature generated using the approach shown in Figure 1(a), the properties of the signature features are described by the parameters used in the creation of the signal as follows.

The cyclic frequency $\alpha$, at which the signature may be detected is

$$
\alpha=\Delta k \Delta f
$$

and the spectral frequency $f_{\text {sig }}$, at which the signature is centred is

$$
f_{\text {sig }}=f_{0}-\left(\frac{K-\Delta k}{2}-k_{\text {sig }}\right) \Delta f
$$

where $\Delta k$ is the separation of the sets of mapped carriers, $\Delta f$ is the separation between adjacent OFDM carriers, $f_{0}$ is the carrier frequency of the OFDM signal, $k_{\mathrm{sig}}$ is the carrier bin index of the central mapped carrier, and $K$ is the total number of IFFT bins used to generate the OFDM signal.

The next section examines the significance of this ability when cyclostationary signatures are used in the context of DSA network frequency rendezvous.

A key consideration in the use of OFDM carrier mapping to generate cyclostationary signatures is the trade off which is made between the strength of the signature generated and the cost in terms of the number of carriers used. The overall number of carriers available for data transmission is reduced by the number of carriers required to carry mapped data symbols and the data rates which may be supported are decreased accordingly. However, although signatures may be generated using carriers solely dedicated to this purpose, it may also be possible to take advantage of signatures created as a side-effect of existing signal components. Figure 3 shows one such case. The normalized SCD illustrated is that of a simulated WiMax IEEE 802.16 OFDM waveform [7]. In the generation of the 256-carrier WiMax OFDM waveform, static carrier bin indices are specified for 8 pilot carriers. On both the uplink and downlink, a number of these carriers are modulated using the same value, derived from a pseudorandom binary sequence generator. This results in the trellis pattern of single carrier signatures which can be seen in the SCD. These signatures may be used themselves to detect and identify WiMax signals in a DSA networking environment or 
alternatively, they may be used to contribute to more powerful signatures formed using additional mapped carriers.

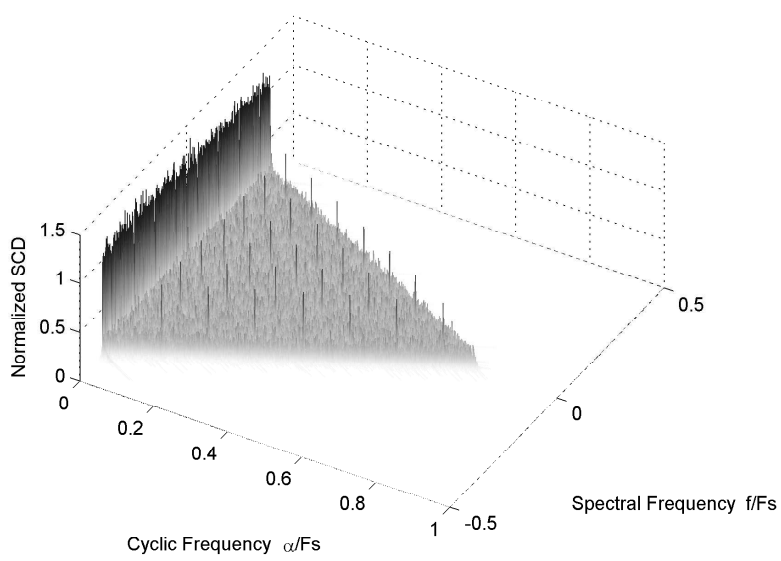

Fig. 3. Normalized SCD of a WiMax 256 bin OFDM waveform showing signatures due to the use of pilot carriers

Section $\mathrm{V}$ examines the trade offs which may be made in the generation of cyclostationary signatures in more detail using experimental results.

\section{B. Cyclostationary Signatures in Dynamic Spectrum Access Networks}

Section II-A examined some of the key benefits of dynamic or decentralized access to radio spectrum. In order for these benefits to be realized, a number of significant challenges must be overcome. One such challenge is that of frequency rendezvous in decentralized DSA networks. In this section, cyclostationary signatures are presented as a mechanism for enabling the establishment and maintenance of communications in situations where operating frequencies and bandwidths may not be predetermined and can change dynamically during the operating lifetime of the network.

DSA network frequency rendezvous refers to the process by which a device may

i) create a network by establishing communications with one or more devices or

ii) detect, classify and synchronize with the signal of peer devices in an existing network

in an environment where the operating frequencies and/or bandwidth may be dynamic variables of the network.

In order to effectively carry out these tasks, a mechanism which permits wide frequency bands to be scanned and rapidly searched for signals of interest is required.

Coherent methods of signal detection and classification can provide optimal performance however they typically require a priori knowledge of the carrier frequencies of the signals of interest and so are unsuitable for this task. Also, coherent techniques are often tailored to the characteristics of the particular signal to be detected and may not be capable of detecting signals whose characteristics vary dynamically during the course of operation. Radiometric schemes provide a low complexity non-coherent technique for signal detection over a frequency band of interest. However, although they may be used to detect a wide range of signal types, they offer an extremely limited ability to extract information about signals which may be present, are sensitive to noise level variations and do not perform well in situations where more than one signal exists in the frequency band being analyzed.

Cyclostationary signal analysis offers the ability to detect and classify signals with levels of performance approaching those of optimal coherent schemes [25] without the phase information required by those approaches and maintains the generality of other non-coherent approaches while overcoming their main limitations. Cyclostationary signatures provide a highly flexible technique which allows the advantages of cyclostationary signal analysis to be leveraged in fulfilling the requirements of frequency rendezvous in DSA networks.

The first of these requirements is the ability of a device within a DSA network to detect a similar device or network of devices which may be operating within its power and frequency range.

As shown in Figure 1, a cyclostationary signature may be added to a signal in such a way as to cause a distinctive peak at a single value of $\alpha$ in the SCD of the signal. Using cyclostationary analysis, this peak may be used to detect the signal even at low signal-to-noise ratios (SNR). As this signature is created in the frequency domain through the use of IFFT bin mapping, it is continuously present in the time domain signal and may be detected by analyzing any segment of a signal received from any device using a particular signature. Although the performance of a full SCD analysis of a received signal may be computationally complex, the presence of the cyclostationary signature at a single $\alpha$ row of the SCD permits a much more efficient approach to be used. This approach involves the use of a time-smoothed cyclic cross periodogram which is calculated over the $\alpha$ row of interest and a second reference row and is discussed in detail in the next section.

The second requirement of network rendezvous is the ability to classify a detected signal of interest. In this, the flexibility with which cyclostationary signatures may be generated plays an important role. In Section III-A it was shown that the value of the cyclic frequency, $\alpha$ at which the signature may be detected is dependent upon $\Delta k$, the number of IFFT bins between mapped carriers and $\Delta f$, the separation of adjacent carriers of the OFDM waveform. By specifying the OFDM carriers which are to be mapped in a given system, $\Delta k$ may be chosen and the $\alpha$ row location of the signature determined. This ability to generate system-specific signatures may be used to provide an effective signal classification mechanism. A designer may choose a unique signature for devices of a particular system which will differentiate signals of that system from those of others present in the same frequency bands of operation. The signature detection approach mentioned above and outlined in the following section may be tailored to a specific signature $\alpha$ row location and will reject all signals other than those with the signature required. 
Cyclostationary signatures also offer significant advantages in fulfilling the third requirement of network rendezvous in DSA networks - that of signal acquisition. OFDM-based transmission schemes are highly sensitive to frequency offset errors due to the use of multiple closely spaced carriers. The orthogonality of these carriers is preserved as each occurs at a null in the spectrum of those adjacent to it. In the event of a frequency offset, the location of the carriers is shifted from these null positions and Adjacent Carrier Interference (ACI) significantly reduces system performance [26]. Due to this sensitivity, OFDM systems typically employ a twostage approach to carrier frequency synchronization - an initial coarse carrier frequency acquisition stage and a second fine carrier frequency tracking stage [27] [28]. Techniques employed for coarse-frequency acquisition have included the use of training symbols [29], null symbols [30], cyclic prefix correlation [31] as well as cyclostationary analysis [32]. The acquisition range of these techniques is generally on the order of a number of carrier frequency spacings which, although sufficient for non-DSA networking applications, is too narrow for acquisition in an environment where the signal of interest may lie in a frequency band many times the width of the signal itself. The use of cyclostationary signatures permits the acquisition range for OFDM signals to be extended to the width of the RF front-end of the receiver in question. As shown in Section III-A, the carrier frequency $f_{0}$ of an OFDM signal is directly related to the spectral frequency $f_{\text {sig }}$ at which the detected signature is centred. If the parameters used in the generation of the signature are known, its spectral frequency location may be used to estimate the carrier frequency location with sufficient accuracy to then adopt an accurate second-stage tracking technique. Figure 4 shows an OFDM waveform with an embedded signature generated using 5 mapped carriers and centred at the carrier frequency. The carrier frequency may be clearly identified by the distinct peak in the spectral frequency profile of the signal for the signature cyclic frequency $\alpha_{\text {sig. }}$.

\section{EXPERIMENTAL APPROACH}

In Section III, the novel concept of cyclostationary signatures was presented and the advantages of their use for frequency rendezvous in dynamic spectrum access networks were discussed. In this section the experimental approach adopted to examine the performance of cyclostationary signatures when used for frequency rendezvous are outlined.

In order to assess the suitability of cyclostationary signatures when used in the context of dynamic spectrum access frequency rendezvous, a highly reconfigurable platform for cognitive network experimentation known as the Plastic Project [33] was utilized. A reconfigurable and computationally efficient signature detector based on the time-smoothed cyclic cross periodogram was implemented on the platform and experiments using a range of cyclostationary signature types were carried out.

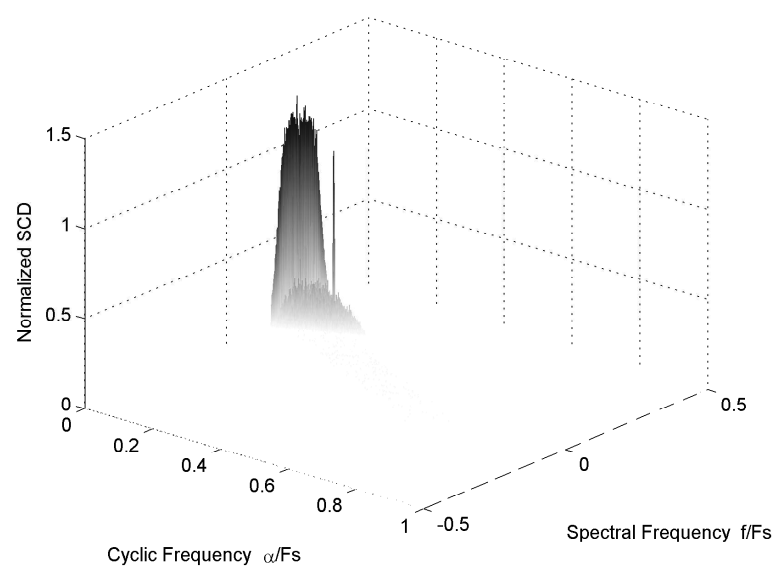

(a) Normalized Spectral Correlation Density

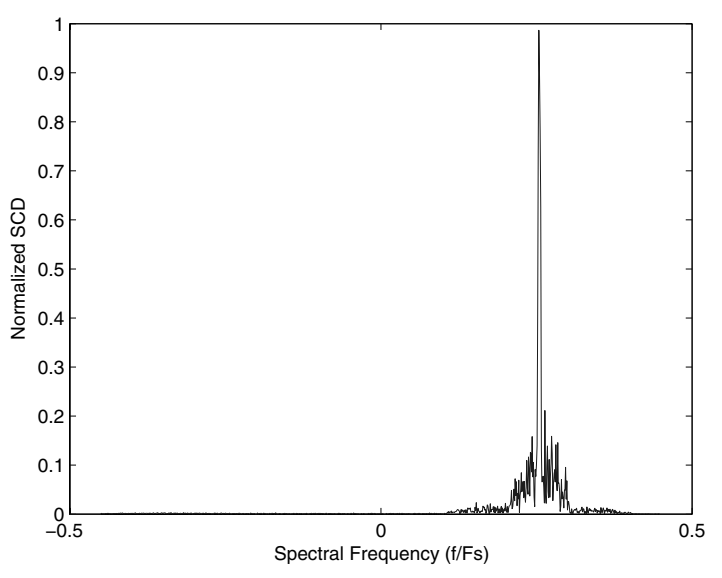

(b) Spectral frequency profile for $\alpha_{\text {sig }}$

Fig. 4. Using the cyclostationary signature location for coarse carrier frequency estimation

\section{A. The Plastic Project - a highly reconfigurable platform for cognitive network experimentation}

A Cognitive Network is comprised of nodes which are capable of observing network conditions, reasoning about their environment and adapting to use the resources available in the most efficient manner possible.

Such nodes require a high degree of reconfigurability at all layers from application to physical as well as mechanisms to support dependencies which occur across these layers. The Plastic Project comprises a network node architecture specifically designed to support the levels of adaptivity required in cognitive networking applications while effectively managing the complexity of such a system through the use of a component based design. A given node configuration consists of a number of heterogeneous hardware and software components which are linked together to form a structure which supports a flow of data between an application and an RF front-end. An XML configuration document is used to specify the manner in which these components are linked and a support structure comprising a document parser, a component 
manager and a node stack manager serves to maintain the flow of data while the system reconfigures in response to given observations and events. The system architecture provides the ability to reconfigure and adapt on each of three specific levels, namely

i) Parametric,

ii) Structural and

iii) Application.

Parametric reconfiguration refers to the reconfiguration of individual parameters within each software or hardware component. These are exposed by the designers of the individual components and may be used to adjust, for example, the centre frequency of an adaptive RF front end, the number of carriers used in an OFDM transceiver or the complexity of an adaptive modulation scheme. Structural reconfiguration consists of the insertion, removal or replacement of any component within the node structure. This approach may be adopted to change the air interface used by the node, to switch from a TDMA to an FDMA based MAC layer or to change from a single to multiple carrier transmission scheme. The highest level of reconfiguration supported is Application reconfiguration and consists of the replacement of the entire node structure with an alternative configuration in order to perform a completely different role. This level of reconfiguration permits the operation of a node to be switched from a subscriber station within an IEEE 802.16 WiMax network to a member of an ad-hoc UWB network for example.

An additional level of component granularity is supported by the Plastic Project at the physical layer through the inclusion of a highly reconfigurable software radio implementation known as IRIS (Implementing Radio in Software) [34]. IRIS runs on a general purpose processor and is in itself a complex component-based architecture. A reconfigurable radio is implemented in IRIS using signal processing units which may be chained together to support a flow of data to and from a hardware front-end. Parameters within these units may be dynamically reconfigured while the radio operates and the units themselves may be inserted, removed and replaced in order to support reconfiguration on each of the three levels discussed above.

Over 100 different reusable signal processing units have been implemented for the IRIS architecture including those implementing multicarrier transmission schemes, filtering, modulation, coding, synchronization and signal analysis. Together with these software units are a number of hardware units used to interface with a range of radio front-end devices.

The following section outlines the implementation of a reconfigurable cyclostationary signature detector as one of the signal processing units within the IRIS component of the Plastic Project.

\section{B. A Reconfigurable Cyclostationary Signature Detector}

A key issue in the use of cyclostationary signal analysis is the computational complexity associated with the calculation of a complete spectral correlation density function [35]. This complexity arises due to the large numbers of complex convolution operations required. In the design of a signature detector to be implemented on a general purpose processorbased software radio architecture, the issue of complexity is especially important.

One advantage of the use of cyclostationary signatures of the type illustrated in Figure 1 is the fact that the signature features are confined to a narrow range on the $\alpha$ axis. This permits the design of a signature detector which requires only a small fraction of the full SCD function to be estimated.

In implementing a suitable signature detector for our platform, a time-smoothed cross cyclic periodogram approach was chosen. This approach permits the estimation of an SCD function over individual values of the cyclic frequency, $\alpha$, and thus allows significant gains in efficiency to be realized. The implementation of the signature detector is illustrated in Figure 5. The Fourier transform stage of the detector was implemented using the highly efficient Fastest Fourier Transform in the West (FFTW) C subroutine library [36]. A sliding window of samples are transformed and then duplicated and shifted to obtain a number of complex demodulates. The complex demodulate values are then multiplied to obtain the SCD estimate at values of $\alpha_{\text {sig }}$ and $\alpha_{\text {nosig. These represent the }}$ SCD function estimated over two $\alpha$ rows - the first containing the cyclostationary signature features and the second free of signature-related features. An average is obtained over a number of data windows and a detection statistic is calculated. The detection statistic, $D_{\alpha}$ is defined as the ratio of the maximum values of these two rows as follows:

$$
D_{\alpha} \doteq \frac{\max S_{x}^{\alpha}(f)_{\text {sig }}}{\max S_{x}^{\alpha}(f)_{\text {nosig }}}
$$

A greater value of $D_{\alpha}$ indicates a greater confidence in the presence of a cyclostationary signature. Finally, a threshold value is used to arrive at a detection decision.

The ability to perform coarse carrier frequency estimation through analysis of the signature properties was discussed in Section III-B and was included as a feature of the detector implementation. As shown in Figure 5, upon detection of a signature, the carrier frequency is estimated through analysis of $S_{x}^{\alpha}(f)_{\operatorname{sig}}$ and is provided as the second output of the detector.

A significant advantage afforded by the use of a reconfigurable software radio platform is the ability to specify reconfigurable parameters in the design of a signal processing algorithm. This approach was used to permit the values of $\alpha_{\text {sig }}$ and $\alpha_{\text {nosig }}$ within the cyclostationary signature detector to be dynamically adjusted during the course of operation. In this way, it was possible to implement a general detector which is not limited to the detection of any specific signature but which may be dynamically reconfigured in order to detect any cyclostationary signatures generated through the use of OFDM carrier mapping.

The performance of the detector implementation is examined using experimental results in Section V. 


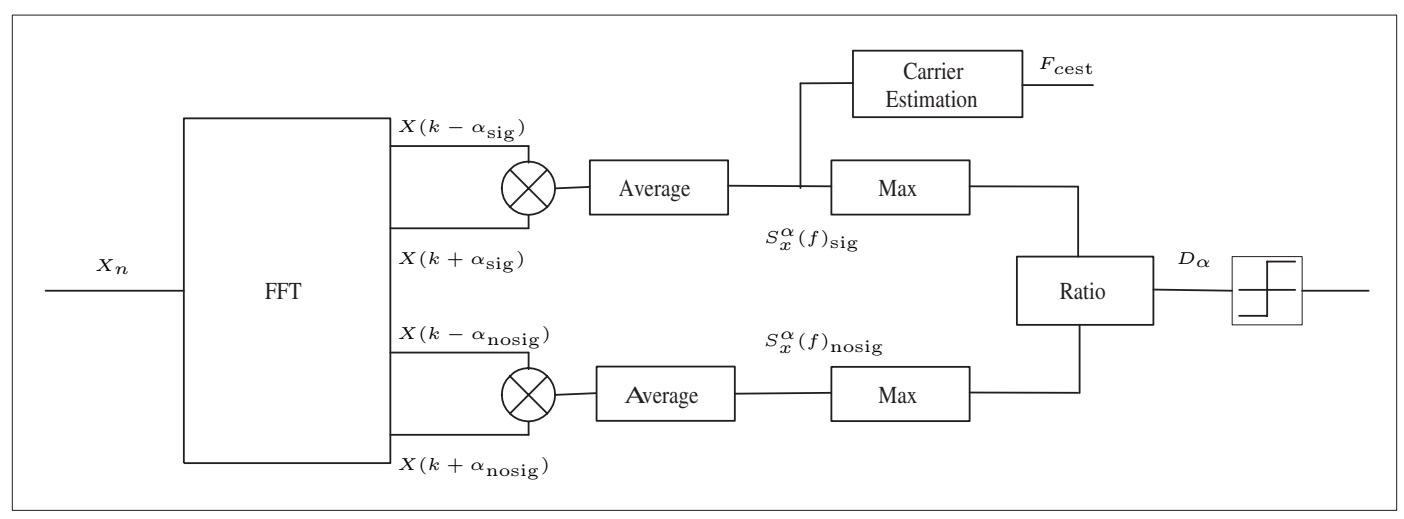

Fig. 5. The Cyclostationary Signature Detector

\section{Cyclostationary Signature Experimentation}

The suitability of cyclostationary signatures for frequency rendezvous and the performance of our signature detector implementation were examined by generating, transmitting, receiving and analyzing suitable signals in the $2.4 \mathrm{GHz}$ ISM band. 256-carrier OFDM signals containing suitable signatures were initially generated using MATLAB. These signals were then transferred to an Anritsu MG3700A Vector Signal Generator and were transmitted at a rate of $1 \mathrm{MHz}$.

In order to receive, detect and analyze the transmitted signals, a cyclostationary signature detector was implemented using the Plastic Project and the IRIS architecture.

A highly reconfigurable RF front-end for software radios known as the Universal Software Radio Peripheral (USRP) was used with our signature detector implementation. The USRP permits complex-valued signals to be transmitted and received over a bandwidth of up to $6 \mathrm{MHz}$ in a range of different frequency bands using a number of daughterboards. Within these frequency bands, the transmit and receive frequencies, data rates and power levels may be adjusted by software over the USB2 connection. Figure 6 shows the USRP and some of the test equipment used. A bandwidth of $4 \mathrm{MHz}$ was sampled using the USRP and analyzed using the signature detector for the presence of signatures at specific values of the cyclic frequency, $\alpha$. Tests were performed using a range of signatures types and detector configurations. The results of these tests are presented and discussed in the following section.

\section{RESUlts}

OFDM signals were generated using QPSK-modulated random data symbols. A 256-bin IFFT was employed and the carrier mapping approach illustrated in Figure 1 was used to embed a cyclostationary signature in each. A number of signals were generated containing signatures formed using 1,5 and 50 OFDM carrier repetitions. These signals were then transmitted at $2.4 \mathrm{GHz}$ and at a rate of $1 \mathrm{MHz}$ using an Anritsu MG3700A Vector Signal Generator. The transmitted signals were received using a USRP RF front-end which was software configured to sample a bandwidth of $4 \mathrm{MHz}$ and were analyzed using the reconfigurable cyclostationary signature

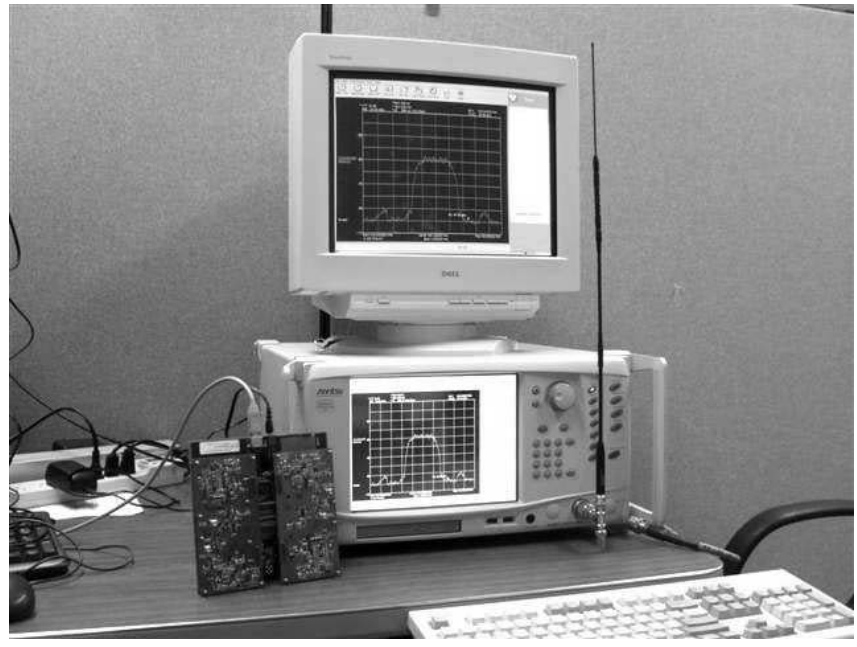

Fig. 6. The USRP and test equipment used for cyclostationary signature experimentation

detector implementation illustrated in Figure 5. The signature detector was implemented in $\mathrm{C}++$ as a signal processing unit of the IRIS software radio physical layer within the Plastic Project architecture.

Initial tests were carried out in order to compare the performance of the detector for signatures of different types over a range of observation times. OFDM signals containing cyclostationary signatures were generated using 1, 5 and 50 repeated carriers. Signals containing no signatures were also generated. These were then transmitted and the output of the detector, $D_{\alpha}$, was recorded for observation times of increasing length. Multiple tests were carried out using each signature type and average values of $D_{\alpha}$ were calculated. The test results are illustrated in Figure 7. An important feature of cyclostationary signal analysis is the improvement in performance which may be acheived through use of a greater observation time. This may be seen in our results as the increasing value of the detection statistic, $D_{\alpha}$.

Results indicate that signatures generated using just a single repeated carrier may be successfully detected through analysis using an adequately large observation time. However, 


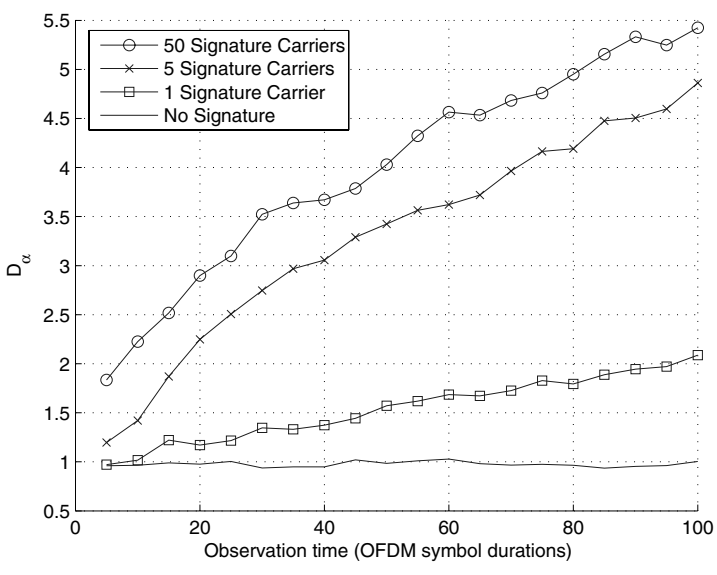

Fig. 7. Detector output, $D_{\alpha}$, observed for signatures generated using 1,5 and 50 OFDM carriers.

signatures generated using 5 repeated carriers were shown to offer the same detection performance over significantly shorter observation times. These performance gains were shown to increase moderately with the number of additional carriers used to generate signatures, albeit at the cost of increased overhead. Table I compares the overhead cost as a percentage of the overall data rate of 256-carrier OFDM for the signatures used. As expected, the output of the detector for signals generated without signatures remains close to unity for all observation times.

TABLE I

COMPARISON OF OVERHEAD ASSOCIATED WITH SIGNATURES FOR 256-CARRIER OFDM

\begin{tabular}{|c|c|}
\hline Carriers Used & \% Overhead Cost \\
\hline 1 & 0.39 \\
5 & 1.9 \\
50 & 19.5 \\
\hline
\end{tabular}

A second set of tests were carried out in order to examine the effect of received SNR on detector performance. A similar approach to that of the previous test was adopted. Signals were generated containing a range of signature types and transmitted at a number of power levels. These signals were received, the SNR was estimated for each, and the output of the detector, $D_{\alpha}$ was recorded for observation times of increasing length. Multiple tests were carried out and average values of $D_{\alpha}$ were calculated.

Figure 8 shows the results observed for signatures generated using 1,5 and 50 carriers. Once again, performance gains were observed in each case for observation times of increasing length.

Results obtained indicate that improvements in detector performance may be achieved through the use of signatures generated using greater numbers of carriers. As was the case in the tests described above, these improvements are most significant when performance for 5-carrier and single-carrier signatures is compared. Although signatures generated using

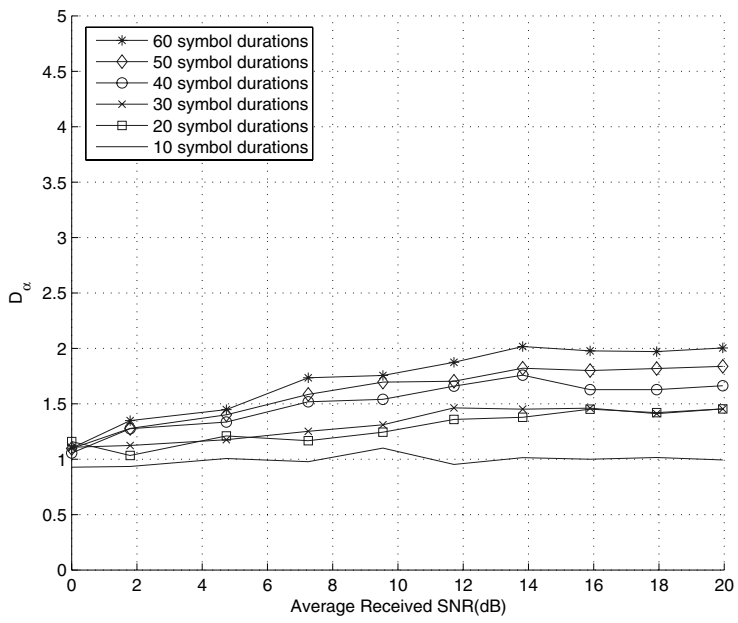

(a) 1 carrier

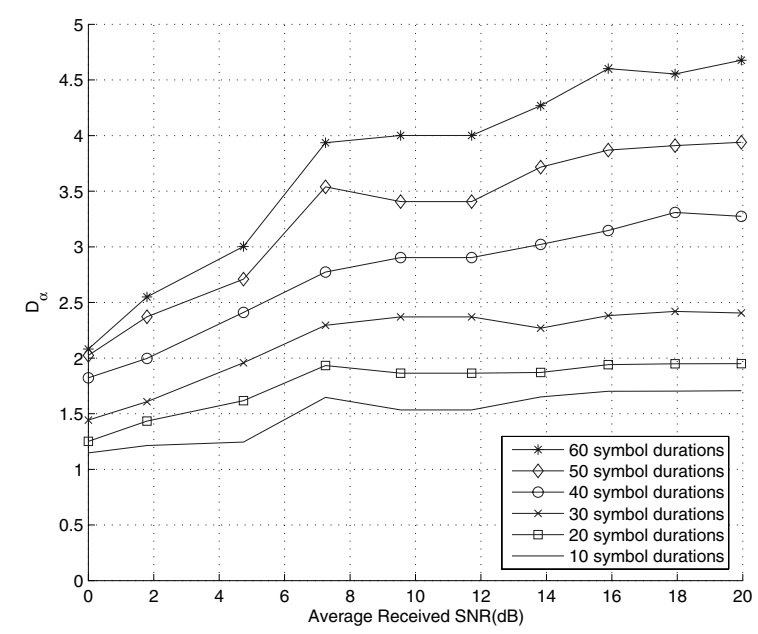

(b) 5 carriers

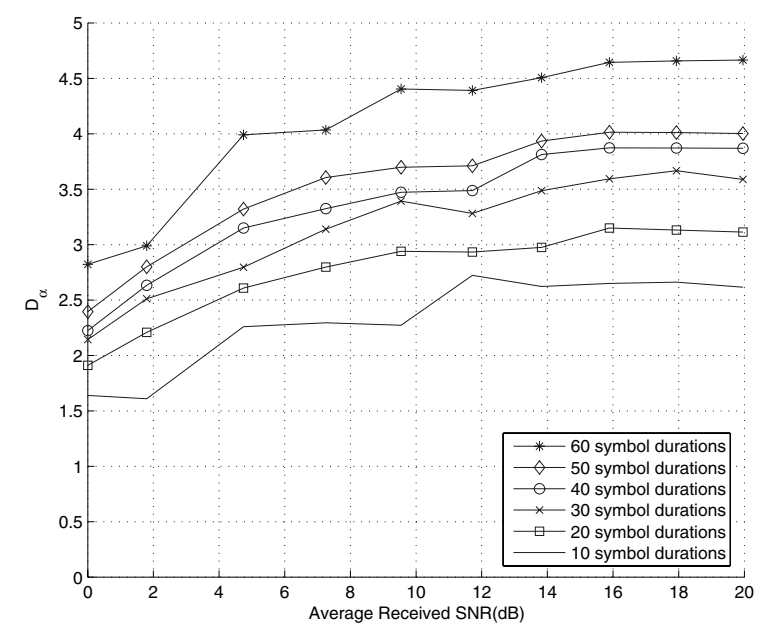

(c) 50 carriers

Fig. 8. Observed SNR performance for signatures generated using 1, 5 and 50 OFDM carriers 
50 carriers offer further performance gains as shown in Figure $8(\mathrm{c})$, these come at the cost of increased overhead.

A key consideration for signature detection is the observation time required by the detector in order to reach a reliable decision. In addition to experiments performed using the Plastic Project, Monte Carlo simulations were carried out to examine the Receiver Operating Characteristics (ROC) of the detector for a range of observation times. Signals were generated using 256-carrier OFDM and detector performance was recorded for observation times of 12,18 and 24 symbol durations. 5-carrier signatures were randomly embedded in a number of the signals and the probability of detection, $P_{\mathrm{d}}$, and probability of false alarm, $P_{\mathrm{fa}}$, were observed over 10000 simulations for each observation time. Results are illustrated in Figure 9 for an AWGN channel and average SNR of $5 \mathrm{~dB}$. The observed results indicate that a 5-carrier signature may be detected with $P_{\mathrm{d}}$ of $91.5 \%$ and $P_{\mathrm{fa}}$ of $2.9 \%$ using an observation time equivalent to just 24 OFDM symbol durations. Performance may be further improved by increasing the observation time of the detector.

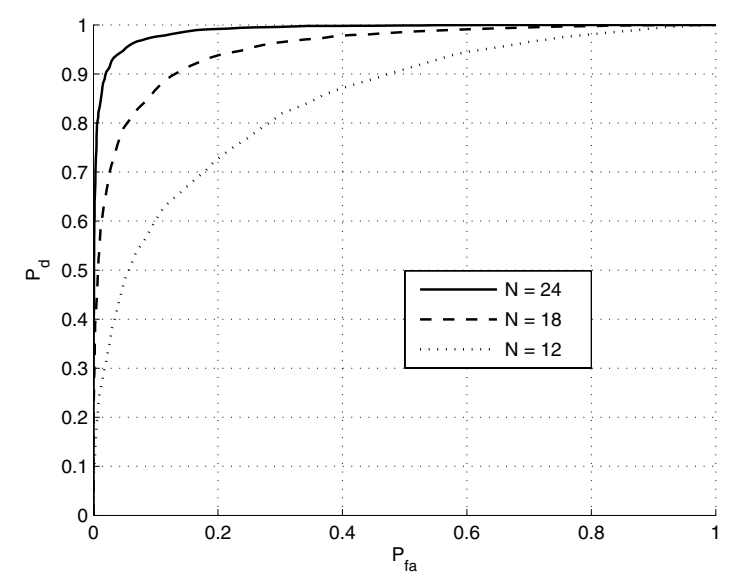

Fig. 9. Detector ROC performance for 5-carrier signatures using observation times of $\mathrm{N}=12,18$ and 24 OFDM symbol durations.

\section{DISCUSSION}

In performing tests using cyclostationary signatures and the Plastic Project platform, a number of key observations were made.

An important consideration in the use of cyclostationary signatures is the additional signaling overhead required. When signatures are generated using OFDM carrier mapping, this overhead arises from the reduction in carriers available for data transmission. The trade off between detector performance and overhead incurred was examined through experimentation with a number of signature types. Results obtained indicate that detectable signatures may be generated using an overhead of just $0.39 \%$ in a 256 -carrier OFDM implementation. However, it was found that significant performance improvements were acheived by incurring an overhead of less than $2 \%$ using a 5-carrier signature.
An important limitation of cyclostationary signal analysis is the long observation time typically required to reliably detect and classify a signal. In the context of a DSA network, this directly impacts the speed with which rendezvous can be achieved. In order to examine the effect of observation time upon signature detection performance, experiments were carried out using the Plastic Project platform. It was found that an observation time of 80 OFDM symbol durations was required in order to reliably detect a single-carrier signature. However, in order to obtain the same performance using a 5-carrier signature, it was found that an observation time of just 15 OFDM symbol durations was required. Observation time effects were further examined using simulations. Results obtained indicate that a $P_{\mathrm{d}}$ of $91.5 \%$ and $P_{\mathrm{fa}}$ of $2.9 \%$ may be acheived using a 5-carrier signature and an observation time of just 24 OFDM symbol durations. An important criterion for DSA network rendezvous is the ability to reliably detect and classify a signal using an observation time of less than a single frame duration. A WiMax signal using a $3.5 \mathrm{MHz}$ channel and a $1 / 8$ cyclic prefix requires a minimum of 34 OFDM symbols per frame. Therefore, a 5-carrier signature may be used to reliably detect and classify a WiMax signal using an observation time of less than a single frame duration.

Continuing from the initial research outlined in this paper, future work will be undertaken to develop MAC and Network layer protocols for DSA systems which take advantage of distributed rendezvous using cyclostationary signatures. These protocols will be developed for the Plastic Project test platform and will explore the benefits of using cross-layer solutions as supported by this platform. In addition, further research will be carried out in the applications of cyclostationary signatures for frequency acquisition and tracking in OFDM systems, development of improved architectures for signature detection and classification and the use of cyclostationary signatures in adaptive networks with increasingly distributed and disaggregated structures.

\section{CONClusions}

In developing techniques for generating, detecting and analyzing cyclostationary signatures for OFDM-based systems and performing tests using transmitted signals with an existing platform, four key points have been proven.

First, it has been shown that cyclostationary signatures offer a novel mechanism for the detection and classification of OFDM-based signals in DSA networks. Second, the use of OFDM carrier mapping has been shown to be a highly flexible technique for the generation of signals containing embedded cyclostationary signatures. Third, it has been demonstrated that a low complexity receiver may be implemented in order to perform detection and classification of signals containing cyclostationary signatures. Finally, it has been shown that cyclostationary signatures offer an effective technique for overcoming the issue of distributed frequency rendezvous in OFDM-based DSA networks.

It was found that the use of the Plastic Project test platform made it possible to rapidly prototype a signature detector 
implementation and carry out tests using real signals. This approach permitted valuable insights to be gained in the practical challenges of using cyclostationary signatures for DSA network frequency rendezvous and enabled a much more rigorous assessment to be carried out than would be possible using simulations alone.

\section{ACKNOWLEDGMENTS}

The authors wish to thank Dr. Charles Bostian and Tom Rondeau of Virginia Tech for the use of test equipment required to perform the experiments described in this paper.

This material is based upon work supported by Science Foundation Ireland under Grant No. 03/CE3/I405 as part of the Centre for Telecommunications Value-Chain Research (CTVR) at Trinity College Dublin, Ireland.

\section{REFERENCES}

[1] McHenry, M and McClosky, D, New York City Spectrum Occupancy Measurements September 2004, Shared Spectrum Company, www.sharedspectrum.com.

[2] Lehr, W. and Crowcroft, J., "'Managing shared access to spectrum", New Frontiers in Dynamic Spectrum Access Networks, 2005. DySPAN 2005. 2005 First IEEE International Symposium on, vol., no.pp. 160- 169, 8-11 Nov. 2005

[3] Buddhikot, M.M. and Kolodzy, P. and Miller, S. and Ryan, K. and Evans, J., "'DIMSUMnet: new directions in wireless networking using coordinated dynamic spectrum", World of Wireless Mobile and Multimedia Networks, 2005. WoWMoM 2005. Sixth IEEE International Symposium on $a$, pp. 78- 85, 13-16 June 2005

[4] Gardner, W.A., Cyclostationarity in Communications and Signal Processing, New Jersey: IEEE Press, 1993.

[5] Kolodzy, Paul, Spectrum Policy Task Force Report, Office of Engineering and Technology, Federal Communications Commission, November, 2002

[6] IEEE Standards Association, Local and Metropolitan Area Network Specific Requirements - Part 11: Wireless LAN Medium Access Control (MAC) and Physical Layer (PHY) Specifications, IEEE 802.11-1999, 1999.

[7] IEEE Standards Association, IEEE Standard for Local and metropolitan area networks Part 16: Air Interface for Fixed Broadband Wireless Access Systems, IEEE 802.16-2004, 2004.

[8] European Telecommunications Standards Institute (ETSI), Radio Broadcasting Systems; Digital Audio Broadcasting (DAB) to Mobile, Portable, and Fixed Receivers, ETSI 300 401, 2001.

[9] European Telecommunications Standards Institute (ETSI), Digital video broadcasting (DVB); Framing structure, channel coding and modulation for terrestrial television, ETSI 300 744, Nov. 2004.

[10] Cordeiro, C. and Challapali, K. and Birru, D. and Sai Shankar, "'IEEE 802.22: the first worldwide wireless standard based on cognitive radios", New Frontiers in Dynamic Spectrum Access Networks, 2005, DySPAN 2005, First IEEE International Symposium on, pp. 328-337, 8-11 Nov. 2005.

[11] Nolan, K.E. and Doyle, L. and Mackenzie, P. and OMahony, D., "Fluid Wireless - Dynamic Spectrum Allocation and Spectrum-Monitoring Application Using Reconfigurable Radio and OFDM"', Proceedings of the 2005 Software Defined Radio Forum (SDR Forum) Technical Conference, Anaheim, CA, USA, November 14-18 2005.

[12] Weiss, T.A. and Jondral, F.K., "'Spectrum pooling: an innovative strategy for the enhancement of spectrum efficiency"', Communications Magazine, IEEE , vol.42, no.3, pp. 8-14, Mar 2004.

[13] Gardner, W.A., "'Exploitation of spectral redundancy in cyclostationary signals"', Signal Processing Magazine, IEEE, vol.8, no.2 pp.14-36, Apr 1991

[14] Gardner, W., "'Spectral Correlation of Modulated Signals: Part I-Analog Modulation", Communications, IEEE Transactions on, vol.35, no.6pp. 584- 594, Jun 1987

[15] Gardner, W. and Brown, W. and Chih-Kang Chen, "Spectral Correlation of Modulated Signals: Part II-Digital Modulation"', Communications, IEEE Transactions on, vol.35, no.6pp. 595- 601, Jun 1987
[16] Gardner, W.A. and Spooner, C.M., "'Signal interception: performance advantages of cyclic-feature detectors"', Communications, IEEE Transactions on , vol.40, no.1pp.149-159, Jan 1992

[17] Cabric, D. and Brodersen, R.W., "Physical layer design issues unique to cognitive radio systems", Personal, Indoor and Mobile Radio Communications, 2005. PIMRC 2005. IEEE 16th International Symposium on , vol.2, no.pp. 759- 763 Vol. 2, 11-14 Sept. 2005.

[18] Sai Shankar N. and Cordeiro, C. and Challapali, K., "'Spectrum agile radios: utilization and sensing architectures," New Frontiers in Dynamic Spectrum Access Networks, 2005. DySPAN 2005. 2005 First IEEE International Symposium on , vol., no.pp. 160- 169, 8-11 Nov. 2005.

[19] Gini, F. and Giannakis, G.B., "Frequency offset and symbol timing recovery in flat-fading channels: a cyclostationary approach," Сотmunications, IEEE Transactions on , vol.46, no.3pp.400-411, Mar 1998.

[20] Heath, R.W., Jr. and Giannakis, G.B., "Exploiting input cyclostationarity for blind channel identification in OFDM systems," Signal Processing, IEEE Transactions on [see also Acoustics, Speech, and Signal Processing, IEEE Transactions on], vol.47, no.3pp.848-856, Mar 1999.

[21] Adlard, J.F., Frequency shift filtering for cyclostationary signals, Ph.D Thesis, University of York, 2000.

[22] Allen, R.L. and Mills, D., Signal Analysis: Time, Frequency, Scale, and Structure, John Wiley and Sons Inc, Hoboken, NJ 07030, USA, 2004.

[23] Bolcskei, H., "Blind estimation of symbol timing and carrier frequency offset in wireless OFDM systems," Communications, IEEE Transactions on, vol.49, no.6pp.988-999, Jun 2001.

[24] Fehske, A. and Gaeddert, J. and Reed, J.H., "A new approach to signal classification using spectral correlation and neural networks," New Frontiers in Dynamic Spectrum Access Networks, 2005. DySPAN 2005. 2005 First IEEE International Symposium on, vol., no.pp. 144- 150, 8-11 Nov. 2005.

[25] Gardner, W.A., "Signal interception: a unifying theoretical framework for feature detection," Communications, IEEE Transactions on, vol.36, no.8pp.897-906, Aug 1988.

[26] Pollet, T. and Van Bladel, M. and Moeneclaey, M., "'BER sensitivity of OFDM systems to carrier frequency offset and Wiener phase noise,"' Communications, IEEE Transactions on , vol.43, no.234pp.191-193, Feb/Mar/Apr 1995.

[27] Luise, M. and Reggiannini, R., "'Carrier frequency acquisition and tracking for OFDM systems," Communications, IEEE Transactions on , vol.44, no.11pp.1590-1598, Nov 1996.

[28] Fazel, K. and Kaiser, S., Multi-Carrier and Spread Spectrum Systems, John Wiley and Sons Inc, Hoboken, NJ 07030, USA, 2003.

[29] Schmidl, T.M. and Cox, D.C., "'Robust frequency and timing synchronization for OFDM," Communications, IEEE Transactions on , vol.45, no.12 pp.1613-1621, Dec 1997.

[30] Nogami, H. and Nagashima, T., "A frequency and timing period acquisition technique for OFDM systems,"” Personal, Indoor and Mobile Radio Communications, 1995. PIMRC'95. 'Wireless: Merging onto the Information Superhighway'., Sixth IEEE International Symposium on , vol.3, no.pp.1010-, 27-29 Sep 1995.

[31] van de Beek, J.J. and Sandell, M. and Isaksson, M. and Ola Borjesson, P., "'Low-complex frame synchronization in OFDM systems,"' Universal Personal Communications. 1995. Record., 1995 Fourth IEEE International Conference on, vol., no.pp.982-986, 6-10 Nov 1995.

[32] Byungjoon Park and Hyunsoo Cheon and Eunseok Ko and Changeon Kang and Daesik Hong, "A blind OFDM synchronization algorithm based on cyclic correlation," Signal Processing Letters, IEEE, vol.11, no.2pp. 83- 85, Feb. 2004.

[33] Sutton, P. and Doyle, L. and Nolan, K.E., "'A Reconfigurable Platform for Cognitive Networks"', Proceedings of the 1st International Conference on Cognitive Radio Oriented Wireless Networks and Communications (CROWNCOM 2006), June 8-10, 2006.

[34] Mackenzie, P, Reconfigurable Software Radio Systems, Ph.D Thesis, University of Dublin, Trinity College, 2004.

[35] Roberts, R.S. and Brown, W.A. and Loomis, H.H., Jr., "CComputationally efficient algorithms for cyclic spectral analysis ," Signal Processing Magazine, IEEE, vol.8, no.2pp.38-49, Apr 1991.

[36] Frigo, M.; Johnson, S.G., "'The design and implementation of FFTW3," Proceedings of the IEEE, vol.93, no.2pp. 216- 231, Feb. 2005. 